\title{
Análisis de la percepción de los clientes respecto a la caliidad del servicio recibido por parte del personal que atiende en las grandes superficies de la ciudad de Ibagué $\dot{e}^{\star}$
}

\author{
Analysis of perception regarding customer service quality received \\ by staff attending in big surfaces in Ibagué city
}

\author{
Germán Rubio Guerrero* \\ Mario Samuel Rodríguez Barrero*** \\ Mario Enrique Uribe Macías ${ }^{* * *}$
}

\section{RESUMEN}

En el presente artículo se describen y analizan los resultados del proyecto de investigación "Identificación y análisis de la percepción de los clientes respecto a la calidad del servicio recibido de parte de las grandes superficies de la ciudad de Ibagué", principalmente los relacionados con el personal que presta sus servicios en estos sitios de compra. La investigación es de tipo descriptivo y su objetivo es identificar la percepción de los clientes de las grandes superficies de la ciudad de Ibagué con respecto a la calidad en el servicio al cliente y los factores que la determinan, en el cual los clientes fueron considerados como la unidad de análisis, de los cuales se extrajo una muestra de 380 usuarios. Para el desarrollo del trabajo de investigación, se planteó una estructura teórica y conceptual con el fin de identificar las variables relacionadas con el fenómeno de estudio, y teniendo en cuenta estas variables se diseñaron instrumentos de recolección de datos que permitieron consolidar información relevante, con la cual se presentó un análisis sobre los resultados obtenidos, parte de los cuales se socializan en el presente artículo. Como parte de la investigación, se realizó un análisis sobre la percepción de los usuarios de las grandes superficies con respecto a la calidad del servicio al cliente; se analizaron los factores que determinan la satisfacción o insatisfacción clientes y se evidenció que el servicio que presta el personal encargado de la atención al cliente es un aspecto crítico en la evaluación de la calidad del servicio.

Palabras claves: servicio al cliente, satisfacción de usuarios, percepción del cliente, calidad del servicio en grandes superficies.

\section{ABSTRACT}

In this article are described and analyzed the results of the research project "Identificación y análisis de la percepción de los clientes respecto a la calidad del servicio recibido de parte de las grandes superficies de la ciudad de lbagué", mainly those related to personnel providing services in these places of purchase. The research is descriptive and its objective is to identify the perception of customers from large areas of the city of lbague with respect to the quality of service to the client and the factors that determine it, in which customers were considered as the unit of analysis, from which was extracted a sample of 380 users. For the development of the research work, he was raised as a theoretical and conceptual structure in order to identify the variables related to the phenomenon of study,

* Este artículo es resultado de la investigación "Análisis de la percepción de la calidad del servicio recibido y propuesta de un modelo de gestión de calidad en el servicio al cliente, orientado a las grandes superficies de la ciudad de lbagué", el cual fue desarrollado por los docentes Germán Rubio Guerrero y Mario Enrique Uribe Macías como integrantes del Grupo de investigación GIDEUT de la Universidad del Tolima. Recibido 15 de diciembre de 2012. Aprobado 15 de febrero de 20

* Magister en Administración, Especialista en Gerencia de producción, Especialista en Estadística, Administrador de empresas, Profesor asociado Facultad de Ciencias económicas y administrativas de la Universidad del Tolima, Director del grupo de investigación GIDEUT - Correo electrónico grubio@ut.edu.co

*** Administrador de Empresas. Especialista en Gerencia de Mercadeo. Docente catedrático Facultad de Ciencias económicas y administrativas de la Universidel Tolima - Correo electrónico msrodriguezb@ut.edu.co

*.** Magíster en administración, Especialista en evaluación social del proyectos, Especialista en administración financiera, Administrador de empresas, Profesor asociado Facultad de Ciencias económicas y administrativas de la Universidad del Tolima, Investigador miembro de los grupos de investigación GIDEUT y ECO. Correo electrónico: meuribem@gmail.com 
and taking into account these variables were designed instruments for collecting data that allowed to consolidate relevant information, which presented an analysis on the results, part of which are socialized in this article. As part of the investigation, an analysis on the perception of the users of large areas with regard to the quality of service to the customer; customers were analyzed the factors that determine the satisfaction or dissatisfaction and was evident that our staff's attention to customer service is a critical aspect in the evaluation of the quality of the service.

Keywords: customer service, user satisfaction, customer perception, quality of service in big surfaces Clasificación JEL: L8, L89

\section{INTRODUCCIÓN}

Los servicios constituyen hoy en día el sector de mayor crecimiento en el mundo; desde hace ya varias décadas éste se ha convertido en un factor fundamental de crecimiento y en un elemento clave para la competitividad de las naciones; de igual forma, el proceso de globalización y el fácil acceso a la información han permitido que los clientes de hoy sean personas bien informadas, por lo tanto tienen muchas opciones para elegir qué, cuándo, dónde y cómo satisfacer sus necesidades y deseos.

Entendiendo como servicio el conjunto de prestaciones que el cliente espera recibir además del producto o servicio básico como consecuencia del precio, imagen y reputación del mismo, es un factor que agrega valor al cliente (Uribe, 2010, página 78), se puede decir que el servicio al cliente es también un producto en sí, que puede determinar la fidelización del cliente dependiendo de su satisfacción, siendo de gran importancia su análisis, medición, evaluación y mejoramiento, lo cual también es objeto de análisis en el presente artículo.

En Ibagué, las grandes superficies han tenido un gran auge durante la última década, por tal motivo es importante conocer y analizar la calidad del servicio al cliente en estas organizaciones, identificando desde la óptica del cliente cuáles son los factores críticos y su influencia en la satisfacción de los usuarios. Es así como en este artículo, basado en la investigación "Identificación y análisis de la percepción de los clientes respecto a la calidad del servicio recibido de parte de las grandes superficies de la ciudad de Ibagué", se socializan y discuten parte de los hallazgos de esta investigación, principalmente los relacionados con el servicio prestado por parte del personal que tiene contacto con los clientes, pues a través de dicha investigación, se determinó que el servicio que prestan estas personas, es un punto crítico en la satisfacción de los usuarios.

Después de analizar los resultados mencionados anteriormente, se presentan algunas conclusiones y recomendaciones relacionadas con el servicio al cliente para el mejoramiento de la calidad del servicio en las grandes superficies de la ciudad de Ibagué, las cuales también pueden servir como referencia a las organizaciones con características similares.

\section{DESARROLLO DEL ARTÍCULO}

\section{Marco referencial}

Las grandes superficies son lugares de alta concurrencia por parte de los ibaguereños, quienes buscan satisfacer sus necesidades y deseos a través de los productos y servicios que allí se ofrecen, esperan vivir experiencias agradables y encontrar una buena atención, respaldados por la marca y prestigio de estos sitios de compra. Sin embargo, en ocasiones estas experiencias no son agradables, pues existe inconformidad por parte de algunos clientes debido a las largas filas que deben hacer para pagar, la mala atención del personal, las fallas en el sistema, el incumplimiento en algunos anuncios y el mal servicio de asesoría, postventa y garantías. Estos inconvenientes convierten muchos momentos de verdad en experiencias negativas para los clientes $y, a$ pesar de esto, no se tiene conocimiento de que las grandes superficies cuenten con sistemas de retroalimentación para identificar y corregir estas falencias ni tampoco se perciben estrategias que permitan conocer y mejorar la percepción de los clientes en cuanto al servicio que reciben.

Las grandes superficies cuentan con sistemas de calidad certificados, sin embargo los usuarios no perciben que estos sistemas estén asociados con la calidad en el servicio que reciben, pues no se tiene conocimiento de medios y sistemas que permitan medir la satisfacción desde el punto de vista del cliente, por lo cual, a través de este artículo se presenta la opinión de los usuarios con relación a los aspectos más relevantes y su grado de satisfacción, principalmente en lo relacionado con el personal que atiende a los clientes.

Los usuarios son quienes pueden y deben evaluar la calidad del servicio a través de sus experiencias, por lo cual en primer lugar se define la experiencia como la percepción que cada cliente se forma sobre el servicio que recibe, pues dependiendo de su vivencia única o de una situación repetida, podrá determinar si recibió lo que esperaba. Posee experiencia quien ha conocido una realidad existencial, pues la experiencia es la captación de lo real a través de las facultades sensitivas de conocimiento. Seguidamente, se define también la calidad como el conjunto de características que posee un producto o servicio así como su capacidad de satisfacer los requerimientos del usuario, el cual debe 
cumplir con las funciones y especificaciones para las que ha sido diseñado y debe ajustarse a las necesidades de los clientes (Cuatrecasas, 2010, página 17). La calidad implica satisfacer las necesidades y deseos de los usuarios cumpliendo con los requerimientos del producto o servicio, superando las expectativas del cliente y haciendo desde la primera vez bien lo que haya que hacer.

La norma ISO 8402 define la calidad como el conjunto de características de una entidad que le confieren la aptitud para satisfacer las necesidades establecidas e implícitas (NTC ISO 8402:1986, página 1). Es la conformidad con los requisitos y la excelencia con que se cumplen las expectativas del cliente, entendiéndose como el grado de satisfacción del cliente al adquirir un producto. Los conceptos recientes sobre la calidad, incorporan además la satisfacción de las necesidades y deseos del cliente de una forma adecuada, evaluándola de acuerdo a sus expectativas. La norma ISO 9000:2000 (página 15) conceptúa que la calidad es el grado en el que un conjunto de características inherentes al producto, cumple con las necesidades o expectativas establecidas.

En la versión más reciente de la Norma Internacional ISO (9001:2008, página 22) se considera que la satisfacción del cliente es una de las medidas de desempeño del sistema de gestión de la calidad, por lo cual la organización debe realizar seguimiento de la información relativa a la percepción del cliente. Este seguimiento puede incluir la obtención de elementos de entrada de fuentes como las encuestas de satisfacción del cliente, entre otras. A continuación, figura 1, se muestra el flujograma del Modelo de sistema de gestión de la calidad basado en la satisfacción del cliente.

Figura 1. Modelo de un sistema de gestión de la calidad basado en procesos
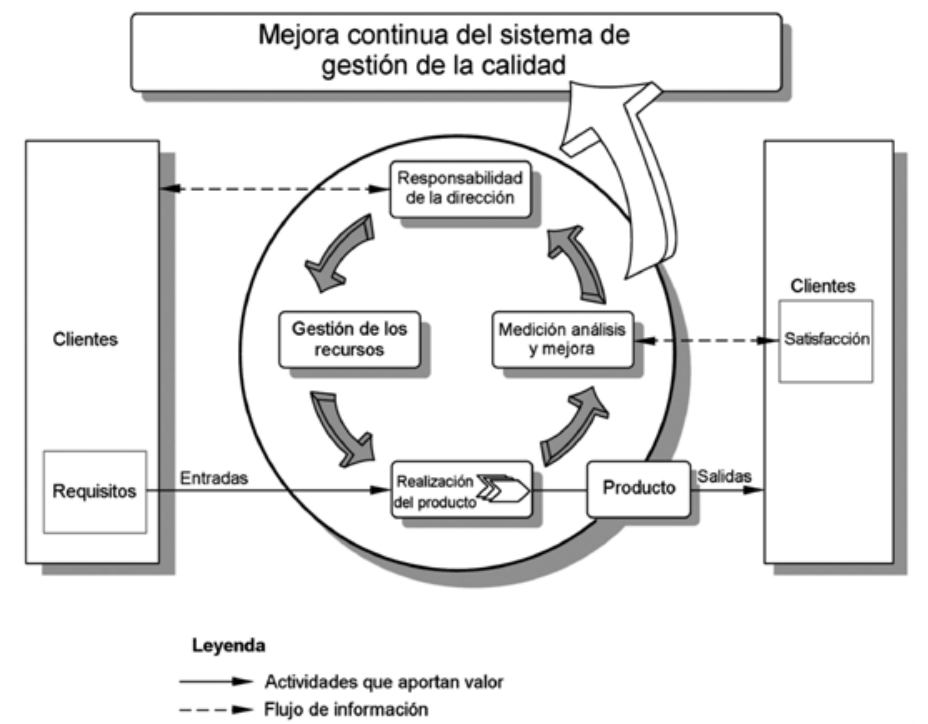

Fuente: Norma ISO 9001:2008, página 7.
De igual forma, el servicio al cliente constituye una fuente de respuestas a las necesidades del mercado y debe surgir mediante la comunicación con el mismo cliente orientando el servicio a la satisfacción de sus necesidades; las organizaciones deben contemplar una estrategia dinámica que les permita prestar un servicio adecuado para lo cual se proponen cuatro condiciones: Interés en el contacto, capacidad resolutiva, flexibilidad y reparación de errores (Ginebra, 1991, página 17 ), pues a través de investigaciones realizadas en Europa y Estados Unidos se ha podido establecer que no se tiene identificado al $96 \%$ de los clientes insatisfechos, por cada queja que se recibe hay 26 clientes con problemas y 6 de estos problemas son graves (Uribe, 2010, página 126). Éstas y otras estadísticas confirman la importancia de identificar la percepción del servicio al cliente desde su propia óptica y muestran la necesidad de crear sistemas de retroalimentación eficaces que permitan realizar procesos de mejora enfocados al cliente, entendiendo que la evaluación que el cliente hace acerca del servicio recibido, se basa en el servicio esperado o expectativas de servicio y el servicio adecuado o mínimo aceptable, entre ambas condicionantes de evaluación existe un rango de tolerancia que separa el servicio esperado del considerado aceptable, de manera que el cliente evalúa su satisfacción en este rango (Montecinos, 2006, página 10).

La satisfacción del cliente se considera como el grado en que se cumplen sus expectativas con relación a un producto y la percepción sobre el grado en que se cumplen sus requisitos (Norma ISO 9000:2000, página 16), depende además del resultado de las percepciones a lo largo de la recepción del servicio menos las expectativas que el cliente tiene al entrar en contacto con la organización (Huete, 2001; citado por Montecinos, 2006 página 194), por lo cual se puede decir que esta satisfacción está sujeta a la evaluación del uso o consumo del producto o servicio por parte del cliente (Oliver, 1993, página 460). Por lo anterior, se concluye que el servicio al cliente es un proceso multidimensional, dinámico y complejo que incluye actividades cognitivas, afectivas y conductuales. La satisfacción del cliente es entonces el resultado de la evaluación de las variables mencionadas, entre las cuales se incluye la confirmación subjetiva, las expectativas, la equidad, el cumplimiento del producto y los resultados de la atribución. De lo anterior se concluye que es importante identificar y analizar la percepción del cliente para medir la calidad del servicio y el desempeño de la organización.

También es importante conceptualizar algunos aspectos relacionados con la investigación, como la atención al cliente, la cual se considera una variable subjetiva, entendida como la percepción que el usuario tiene por los servicios recibidos por parte del personal que lo atendió durante la compra; en ocasiones, esta evaluación se ve afectada por factores que no necesariamente dependen de una persona que labora en el sitio de compra, tales como la ubicación de un producto (layout), un precio errado y/o el manejo de las 
estrategias comunicacionales que utiliza la compañía, sin embargo la claridad, agilidad y efectividad en la respuesta que se dan al cliente, son significativas al momento de evaluar la atención que ellos reciben.

Se conceptúan también los servicios de garantía y posventa como aquellos a los cuales el cliente tiene derecho después de realizar la compra. La calificación de este aspecto depende en gran parte de la claridad de la información que se haya suministrado en el momento de la compra, pues aunque el cliente no da la importancia debida a estas reglas al momento de realizar la compra y la ignora aun después de haberlas escuchado, es importante dejar esta información por escrito y hacer énfasis en los aspectos más relevantes, con base en los reclamos que se presentan con mayor regularidad.

Adicionalmente, se considera la tarjeta de compras como una estrategia de fidelización, la cual ha sido implementada por algunas de las grandes superficies (como la tarjeta de puntos), con la cual muchos clientes han podido acceder a diferentes beneficios tales como créditos para la compra de productos, participación en eventos, rifas, concursos, descuentos, entre otros. Sin embargo, como se analizará más adelante, las dificultades más frecuentes con este servicio tienen relación con fallas en la comunicación, el cobro de altos intereses y/o las cuotas de manejo.

\section{DISEÑO METODOLÓGICO}

La investigación en la que se fundamenta el presente artículo es de carácter descriptivo, en ésta se consideró como unidad de análisis a los clientes de las grandes superficies de la ciudad de lbagué y a través de ellos se buscó determinar la calidad en el servicio y el grado de satisfacción que obtienen al entrar en contacto con dichas organizaciones. Igualmente, para efectos de la investigación se consideraron dos tipos de población, la primera conformada por los clientes que concurren a realizar sus compras en los principales supermercados de las grandes superficies de la ciudad de lbagué y el segundo tipo de población la conformada por las grandes superficies con más de cinco puntos de pago $^{1 *}$. Para el primer tipo de población la selección de los elementos muestrales se realizó por el método de muestreo no probabilístico por conveniencia y juicio del investigador, en razón a que no era posible identificar y seleccionar de manera aleatoria cada unidad. Para el segundo tipo de población, se tomó la sucursal principal de cada una de las grandes superficies. En total se recolectó información a través de 380 clientes en seis sitios de compra, con base en una guía de encuesta previamente diseñada, además se observaron en estos mismos sitios, procesos tales como el ingreso y salida del parqueadero, el proceso de compra

* Durante la investigación se tuvieron en cuenta los supermercados Carrefour, Éxito, Mercacentro, Makro, Homecenter y Yep. y pago, la atención a los clientes y el servicio de asesoría y postventa. En primer lugar se aplicaron las encuestas, y luego se realizaron las observaciones para determinar con mayor exactitud los factores relacionados con la satisfacción o insatisfacción de los clientes por el servicio recibido en las grandes superficies de la ciudad de Ibagué.

\section{IDENTIFICACIÓN DE VARIABLES}

Para identificar las variables que desde la perspectiva del cliente determinan la calidad en el servicio en las grandes superficies de la ciudad de lbagué, durante la investigación se consultó entonces a 380 los clientes sobre los motivos que tenían en cuenta para seleccionar su sitio de compra. Como respuesta a esta pregunta se obtuvieron 508 observaciones, dado que muchos clientes identificaron más de un motivo, los cuales una vez tabulados se agruparon en 15 aspectos principales que se presentan en la tabla 1 de resumen.

De acuerdo a estos resultados, se puede decir que los principales motivos por el cual los clientes escogen su sitio de compra son la economía, la cercanía, la variedad u oferta de productos y/o servicios y la percepción de calidad que tienen con respecto a los productos que allí se ofrecen. También se encontró que los aspectos menos relevantes para los clientes al momento de escoger su sitio de compra son el horario, el parqueadero y el servicio de garantía (contenidos en el aspecto "Otros"), lo cual no quiere decir que estos servicios no sean importantes sino que los usuarios dan por hecho que al escoger su sitio de compra, lo van a encontrar abierto y van a contar con el servicio de parqueadero y garantía si lo requieren.

Posteriormente, se les consultó sobre el nivel de importancia que para ellos tiene cada una de las variables identificadas. Según estos resultados, se determinó que para los clientes encuestados los aspectos más importantes en su orden son: La calidad de los productos, la amabilidad del personal y la atención al cliente, mientras que aspectos como los servicios adicionales, el parqueadero y la publicidad, fueron calificados con menor importancia. Esto no quiere decir que los aspectos con menor peso porcentual no sean importantes para los clientes, sino que en muchos casos los clientes pueden dar por hecho que al llegar a su sitio de compra van a encontrar servicios adicionales, un lugar para parquear, un horario adecuado y facilidades de pago.

Se puede interpretar que al dar una mayor importancia a aspectos como la agilidad en el servicio, la accesibilidad y la atención, los clientes están manifestando su inconformidad en estos aspectos, pues sus expectativas no siempre se cumplen, debido a demoras, dificultades en el acceso y/o mala atención. En la tabla 2 se observan los resultados ponderados, de acuerdo a la asignación porcentual asignada durante la investigación. 
Tabla 1. Motivos para seleccionar el lugar de compras

\begin{tabular}{|c|c|c|c|c|c|}
\hline & & Frecuencia & Porcentaje & $\begin{array}{l}\text { Porcentaje } \\
\text { válido }\end{array}$ & $\begin{array}{l}\text { Porcentaje } \\
\text { acumulado }\end{array}$ \\
\hline \multirow[t]{17}{*}{ Válidos } & Variedad de productos & 80 & 15,7 & 15,8 & 15,8 \\
\hline & Economía & 121 & 23,8 & 23,9 & 39,6 \\
\hline & Calidad de productos & 55 & 10,8 & 10,8 & 50,5 \\
\hline & Cercanía o ubicación & 102 & 20,1 & 20,1 & 70,6 \\
\hline & Comodidad & 42 & 8,3 & 8,3 & 78,9 \\
\hline & Ofertas y promociones & 29 & 5,7 & 5,7 & 84,6 \\
\hline & La atención al cliente & 15 & 3,0 & 3,0 & 87,6 \\
\hline & Regionalismo & 7 & 1,4 & 1,4 & 89,0 \\
\hline & Garantía y postventa & 3 & 0,6 & 0,6 & 89,5 \\
\hline & Servicio & 12 & 2,4 & 2,4 & 91,9 \\
\hline & Tarjeta del supermercado & 4 & 0,8 & 0,8 & 92,7 \\
\hline & Facilidades de pago & 4 & 0,8 & 0,8 & 93,5 \\
\hline & Horario de atención & 1 & 0,2 & 0,2 & 93,7 \\
\hline & Parqueadero & 3 & 0,6 & 0,6 & 94,3 \\
\hline & Grandes cantidades & 20 & 3,9 & 3,9 & 98,2 \\
\hline & $\mathrm{Ns} / \mathrm{Nr}$ & 9 & 1,8 & 1,8 & 100,0 \\
\hline & Total & 507 & 99,8 & 100,0 & \\
\hline Perdidos & Sistema & 1 & 0,2 & & \\
\hline Total & & 508 & 100,0 & & \\
\hline
\end{tabular}

Fuente: Información de los autores

Tabla 2. Importancia de los aspectos evaluados durante la compra

\begin{tabular}{|l|c|c|c|c|c|}
\hline \multirow{3}{*}{\begin{tabular}{l} 
Variable a evaluar \\
\cline { 2 - 6 }
\end{tabular}} & \multicolumn{3}{|c|}{ Cálculo de nivel de importancia relativa basado en las respuestas de los clientes } \\
\cline { 2 - 6 } & No es importante & Es importante & $\begin{array}{c}\text { Es muy } \\
\text { importante }\end{array}$ & $\begin{array}{c}\text { Índice } \\
\text { importancia }\end{array}$ & $\begin{array}{c}\text { Nivel } \\
\text { importancia }\end{array}$ \\
\cline { 2 - 6 } & $0 \%$ & $50 \%$ & $100 \%$ & $\sum(50 \%)+(100 \%)$ & $\begin{array}{c}\text { Índice Importancia } \\
380\end{array}$ \\
\hline Calidad de los productos & 3 & 112 & 265 & 321 & $84 \%$ \\
\hline Amabilidad del personal & 6 & 111 & 262 & 318 & $84 \%$ \\
\hline La atención al cliente & 10 & 112 & 258 & 306 & $83 \%$ \\
\hline Agilidad en la atención & 17 & 115 & 248 & 303 & $80 \%$ \\
\hline Economía & 13 & 123 & 241 & 298 & $78 \%$ \\
\hline Surtido: marcas y tamaños & 11 & 140 & 228 & 285 & $75 \%$ \\
\hline Claridad en la información & 17 & 157 & 206 & 280 & $74 \%$ \\
\hline Garantía y servicio posventa & 25 & 140 & 210 & 278 & $73 \%$ \\
\hline Amplitud de los pasillos & 17 & 170 & 193 & 271 & $73 \%$ \\
\hline Ofertas y promociones & 27 & 150 & 203 & 261 & $71 \%$ \\
\hline Orientación y señalización & 30 & 158 & 192 & 261 & $69 \%$ \\
\hline Cercanía & 39 & 160 & 181 & 251 & $69 \%$ \\
\hline Horario de atención & 36 & 166 & 178 & 223 & $66 \%$ \\
\hline Facilidades de pago & 46 & 164 & 169 & 219 & $59 \%$ \\
\hline Publicidad & 74 & 161 & 142 & 201 & $58 \%$ \\
\hline Accesibilidad al parqueadero & 65 & 160 & 139 & & $53 \%$ \\
\hline Servicios adicionales & 27 & 148 & 127 & & \\
\hline
\end{tabular}

Fuente: Información de los autores 
Tabla 3. Nivel porcentual de satisfacción de los clientes

\begin{tabular}{|c|c|c|c|c|c|}
\hline \multirow{2}{*}{ Variable } & \multicolumn{4}{|c|}{ Resultados ponderados de las alternativas de respuesta } & \multirow{2}{*}{$\begin{array}{c}\text { Nivel } \\
\text { de satisfacción }\end{array}$} \\
\hline & Malo & Regular & Bueno & Excelente & \\
\hline Accesibilidad al parqueadero & 0 & 7,26 & 144,54 & 83 & $62 \%$ \\
\hline Amabilidad del personal & 0 & 14,85 & 158,40 & 90 & $69 \%$ \\
\hline Amplitud de los pasillos & 0 & 13,86 & 158,40 & 92 & $70 \%$ \\
\hline Orientación y señalización & 0 & 9,57 & 176,22 & 69 & $67 \%$ \\
\hline La atención al cliente & 0 & 15,51 & 151,14 & 91 & $68 \%$ \\
\hline Calidad de los productos & 0 & 4,29 & 156,42 & 128 & $76 \%$ \\
\hline Economía & 0 & 17,16 & 165,00 & 74 & $67 \%$ \\
\hline Surtido: marcas y tamaños & 0 & 9,24 & 173,58 & 87 & $71 \%$ \\
\hline Agilidad en la atención & 0 & 28,71 & 148,50 & 50 & $60 \%$ \\
\hline Facilidades de pago & 0 & 9,24 & 158,40 & 96 & $69 \%$ \\
\hline Cercanía & 0 & 10,56 & 126,06 & 151 & $76 \%$ \\
\hline Horario de atención & 0 & 5,28 & 149,82 & 134 & $76 \%$ \\
\hline Ofertas y promociones & 0 & 16,83 & 143,88 & 101 & $69 \%$ \\
\hline Garantía y servicio posventa & 0 & 14,19 & 152,46 & 65 & $61 \%$ \\
\hline Claridad en la información & 0 & 11,88 & 166,98 & 67 & $65 \%$ \\
\hline Publicidad & 0 & 11,22 & 161,04 & 80 & $66 \%$ \\
\hline Servicios adicionales & 0 & 27,06 & 97,68 & 28 & $40 \%$ \\
\hline
\end{tabular}

Fuente: Información de los autores

\section{ANÁLISIS DEL NIVEL DE SATISFACCIÓN DE LOS CLIENTES}

Para identificar la percepción de los clientes con relación al servicio recibido en las grandes superficies, se les consultó a los encuestados cómo evaluaban los aspectos anteriormente mencionados $\mathrm{y}$, de acuerdo a la ponderación utilizada en la investigación, se obtuvieron los siguientes resultados (Tabla 3 ).

Según se observa en estos resultados, aspectos como la cercanía, el horario y la publicidad son bien calificados por los clientes, mientras los servicios adicionales, la agilidad en la atención y el servicio de asesoría, garantía y postventa, reciben la más baja calificación por parte de los clientes encuestados. La atención ofrecida por parte del personal que labora en las grandes superficies (vigilantes, impulsadoras, mercaderistas, cajeros, empacadores, supervisores y personal de servicio), también fue incluida en la evaluación y los resultados muestran que el nivel de satisfacción con relación a este aspecto es bajo, pues cerca del $50 \%$ de los clientes se encuentran inconformes con el servicio que reciben por parte del personal de servicio al cliente, para quienes por la naturaleza de su labor, el cliente es su razón de ser. También se observa un bajo nivel de satisfacción con relación al servicio de los supervisores quienes tienen poco contacto con el cliente y al parecer estos pocos momentos de verdad no son satisfactorios para los usuarios.

Así mismo, al evaluar la satisfacción del cliente con relación al servicio que reciben por parte de vigilantes, impulsadores, mercaderistas, cajeros y empacadores, se encontró que en ninguno de estos cargos se alcanza un $70 \%$ de satisfacción, aunque es de esperarse que los clientes atribuyan a estos funcionarios las fallas en la atención, demoras e inconformismo en general, sobre lo cual sería importante profundizar de manera particular en cada establecimiento. A continuación, tabla 4, se presentan los resultados de la investigación relacionados con este aspecto. 
Tabla 4. Evaluación de satisfacción del cliente - Servicio del personal

\begin{tabular}{|l|c|c|c|c|c|}
\hline \multirow{2}{*}{ Evaluación del personal de servicio } & \multicolumn{4}{|c|}{$\begin{array}{c}\text { Resultados ponderados de las alternativas } \\
\text { de respuesta }\end{array}$} & \multirow{2}{*}{$\begin{array}{c}\text { Nivel de } \\
\text { satisfacción }\end{array}$} \\
\cline { 2 - 5 } & Malo & Regular & Bueno & Excelente & \\
\hline Vigilantes - seguridad & 0 & 4,29 & 158,40 & 67 & $60 \%$ \\
\hline Impulsadores -mercaderistas & 0 & 23,10 & 155,10 & 58 & $62 \%$ \\
\hline Cajeros & 0 & 20,79 & 152,46 & 78 & $66 \%$ \\
\hline Empacadores & 0 & 15,84 & 154,44 & 68 & $63 \%$ \\
\hline Supervisores & 0 & 13,53 & 130,02 & 42 & $49 \%$ \\
\hline Personal servicio cliente & 0 & 11,22 & 134,64 & 47 & $51 \%$ \\
\hline $\begin{array}{l}\text { Nivel de satisfacción del cliente } \\
\text { con relación al personal de servicio } \\
\text { (Promedio ponderado) }\end{array}$ & $59 \%$ & & & & \\
\hline
\end{tabular}

Fuente: Información de los autores

A través de las observaciones realizadas en los diferentes sitios de compra, se identificaron también algunas falencias con relación al servicio prestado por parte del personal, lo cual permite corroborar que en las grandes superficies de la ciudad de lbagué hay oportunidades de mejoramiento en la atención que los empleados brindan a los clientes, entre las cuales se presentan las siguientes:

Cuando los clientes hacen una pregunta el funcionario responde desinteresadamente, sin generar valor agregado.

Hay poco personal disponible para asesoría, no están prestos a asesorar y los clientes deben buscarlos.

El personal de impulso y mercaderistas están en permanente diálogo con sus compañeros de trabajo, no colaboran a los clientes y se limitan a dar información únicamente sobre el producto que venden.

Se nota el afán de algunos empleados cuando atienden a los clientes y les dedican el menor tiempo posible.

Con relación al servicio que los usuarios reciben en las oficinas de atención al cliente, los resultados muestran que solo el $40 \%$ de los consultados habían hecho uso de este servicio, por lo cual la evaluación correspondió a este porcentaje de los encuestados (152 clientes de los 380 consultados). En esta evaluación se tuvieron en cuenta aspectos tales como la facilidad para ubicar el servicio, el profesionalismo en la atención, el interés en su situación, la claridad en la información, la agilidad en la respuesta, y la efectividad en la solución, según se observa los siguientes resultados (Figura 2):

Figura 2. Evaluación del servicio recibido en la oficina de "Atención al cliente"

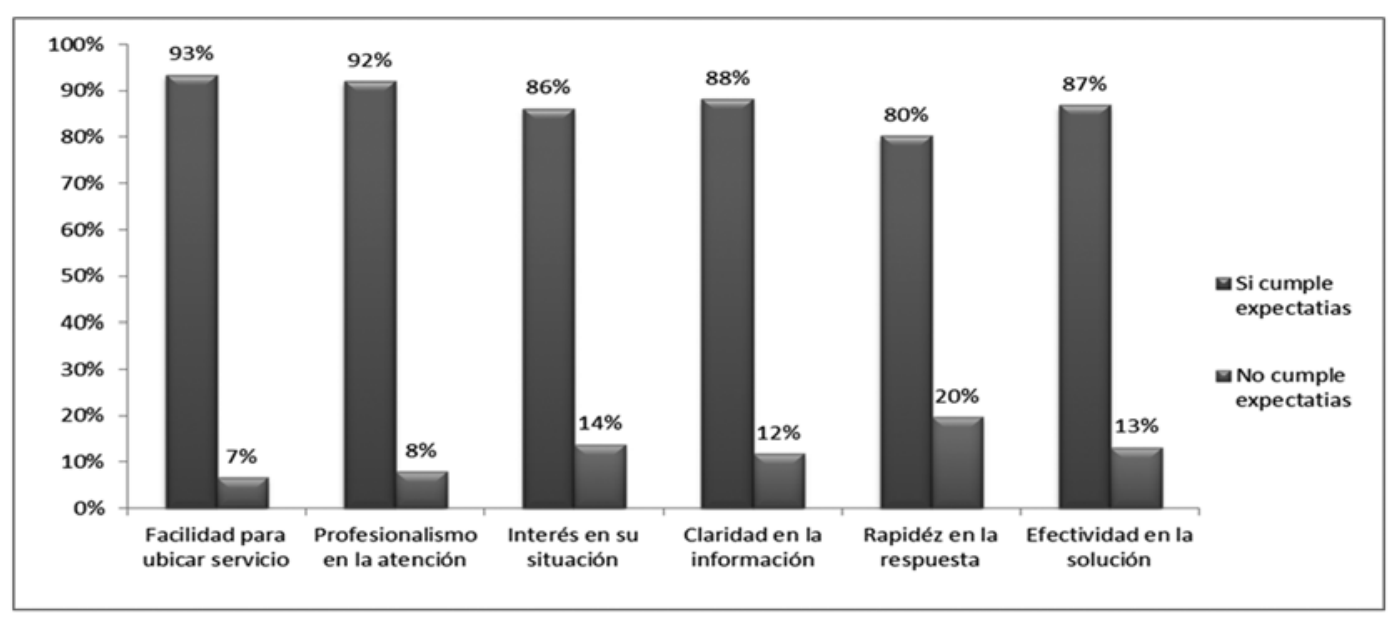

Fuente: Información de los autores 
Los resultados permiten observar un mayor cumplimiento de las expectativas de los clientes con relación a la facilidad que tienen para acceder al servicio y un menor cumplimiento de sus expectativas en relación a la agilidad en la respuesta. En promedio el cumplimiento de las expectativas de los clientes encuestados es del $88 \%$ y un $12 \%$ de no cumplimiento con relación a los servicios que reciben en estas oficinas. Se resalta que la insatisfacción de los clientes se acentúa en aspectos tales como la agilidad en la respuesta, el interés del empleado en la situación del cliente y la efectividad en la solución. Adicionalmente, a través de las observaciones realizadas en las grandes superficies se confirmó que las oficinas de atención o servicio al cliente no siempre están abiertas o disponibles al público, se observó también que en ocasiones están abiertas pero el personal no está disponible para atender a los clientes, bien sea porque están desarrollando otras labores o atendiendo llamadas.

Con el fin de evaluar el servicio de asesoría, garantía y postventa, se hizo inicialmente una pregunta filtro la cual permitió determinar que solo una cuarta parte de los clientes consultados (26\%), había hecho uso de éstos servicios; entonces se consultó solo a estos 97 clientes sobre el cumplimiento de sus expectativas con relación a los servicios de asesoría, garantía y postventa. En estos resultados, como en los anteriores, se observó un alto porcentaje de satisfacción en aspectos como el profesionalismo y la facilidad para ubicar el servicio, pero también se observó un menor grado de satisfacción por la falta de soluciones efectivas y agilidad en la respuesta. En promedio, el cumplimiento de las expectativas de los clientes encuestados con relación a los servicios de asesoría, garantía y postventa es del $83 \%$ y un $17 \%$ el no cumplimiento de dichas expectativas. En la figura 3 se presentan los resultados del cumplimiento de las expectativas de los clientes con respecto a los servicios de asesoría, garantía y postventa

Los resultados de la investigación por superficie, evidencian que existen amplias diferencias en el manejo que cada una de las grandes superficies da a estos servicios, pues mientras en algunas hay un espacio amplio, iluminado y suficientes personas para atender los requerimientos de asesoría, garantía y/o servicio de postventa, en otras estos servicios se prestan en espacios reducidos, casi de manera informal y con escaso personal, que en ocasiones no pertenece a las grandes superficies, sino a empresas proveedoras. De igual forma, se observó que en algunas de estas grandes superficies se dispone de personal capacitado y dedicado a ofrecer asesoría, garantía y/o servicios de postventa, mientras que en otros casos el mismo personal de atención al cliente, supervisores y/o mercaderistas, asesoran a los clientes, lo cual hace que los usuarios perciban que no hay suficiente interés en este tipo de requerimientos. Con relación al servicio de garantía algunos clientes sugirieron que se deben dar soluciones más ágiles, mejorar la información y que la garantía de los productos sea de por lo menos 10 días.

\section{Análisis de la percepción de los clientes}

Para realizar el análisis sobre la percepción de los clientes de las grandes superficies, se plantearon dos preguntas, la primera relacionada con el nivel de satisfacción del servicio recibido y la segunda con relación a la percepción que tenían los encuestados sobre el manejo de sus inquietudes, requerimientos y quejas. A continuación se presenta la figura 4 con los resultados de la primera pregunta.

Figura 3. Evaluación de los servicios de asesoría, garantía y postventa

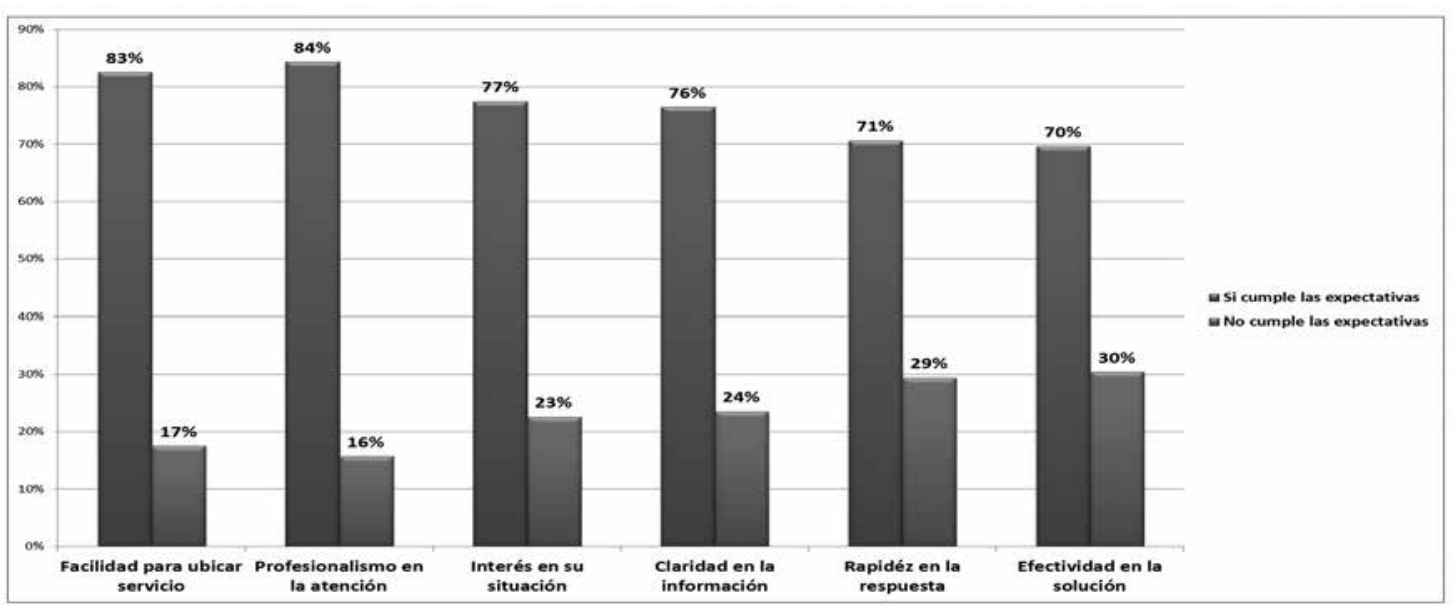

Fuente: Información de los autores 
Figura 4. Nivel de satisfacción de los clientes

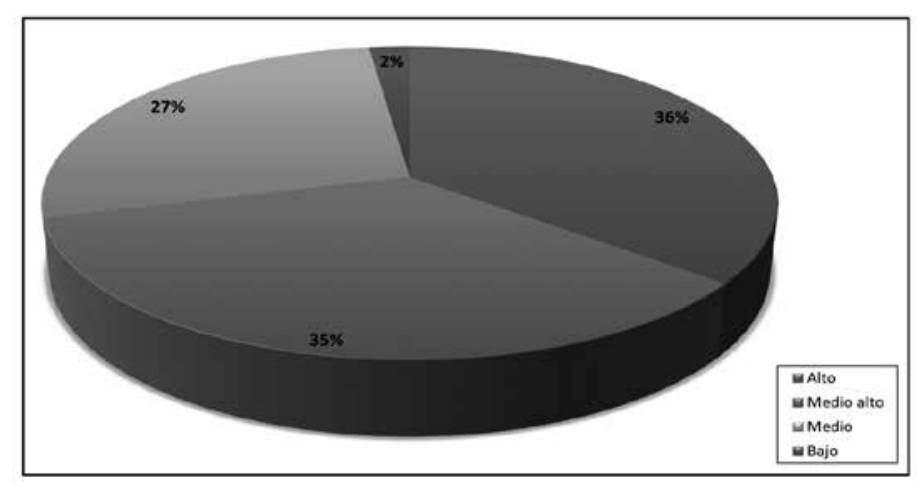

Fuente: Información de los autores

Cabe mencionar en este análisis, que más de cien clientes hicieron observaciones y sugerencias relacionadas con mejoramiento del servicio en las cajas de pago, las instalaciones, la señalización, la garantía, la atención al cliente, el horario, la oferta y calidad de los productos, los precios y promociones, la tarjeta de puntos, el servicio del personal y los servicios adicionales, durante la encuesta. La tabla 5 refleja los resultados ponderados de la evaluación sobre el servicio al cliente.

Tabla 5. Nivel de satisfacción de los clientes, resultados ponderados

\begin{tabular}{|c|c|c|c|c|c|}
\hline \multicolumn{4}{|c|}{$\begin{array}{c}\text { Resultados ponderados } \\
\text { de las alternativas de respuesta }\end{array}$} & \multirow{2}{*}{$\begin{array}{c}\text { Total } \\
\text { puntaje }\end{array}$} & $\begin{array}{c}\text { Nivel de } \\
\text { satisfacción }\end{array}$ \\
\cline { 1 - 3 } Bajo & $\begin{array}{c}\text { Medio } \\
(\mathbf{5 0 \% )}\end{array}$ & $\begin{array}{c}\text { Medio } \\
\text { alto } \\
(\mathbf{7 5 \% )}\end{array}$ & $\begin{array}{c}\text { Alto } \\
\mathbf{( 1 0 0 \% )}\end{array}$ & & \\
\hline 0 & 51,5 & 99,75 & 136 & 287 & $76 \%$ \\
\hline $0 \%$ & $14 \%$ & $26 \%$ & $36 \%$ & & \\
\hline
\end{tabular}

Fuente: Información de los autores

Posteriormente, se consultó a los clientes si conocían los medios para manifestar sus inquietudes, requerimientos y quejas, lo cual dio como resultado que el $53 \%$ de los encuestados si conocía los medios y el $47 \%$ restante, no. También se les preguntó si consideraban que sus requerimientos, sugerencias e inquietudes eran atendidos a lo cual solo el $50 \%$ contesto afirmativamente. Según los resultados de la investigación, los factores por los cuales los clientes consideran que no son atendidos son diversos, sin embargo cabe destacar algunos relevantes como la demora en las respuestas y la baja efectividad en la solución cuando de ello se trata.

\section{CONCLUSIONES}

De acuerdo a los resultados del trabajo de investigación "Identificación y análisis de la percepción de los clientes respecto a la calidad del servicio recibido de parte de las grandes superficies de la ciudad de lbagué", se puede concluir que existen variables que pueden determinar la calidad del servicio al cliente entre las cuales se destacan la amabilidad del personal, la agilidad en la atención, la atención al cliente, la calidad de los productos, las facilidades de pago y los servicios de garantía, asesoría y postventa, variables que fueron objeto de análisis en este artículo.

Con respecto al nivel de importancia que los usuarios dan a cada una de las variables anteriormente mencionadas, se estableció que los aspectos más importantes en su orden son: La calidad de los productos, la amabilidad del personal y la atención al cliente. También se determinó que al dar los clientes una mayor importancia a aspectos como la agilidad en el servicio, la accesibilidad y la atención, están manifestando su inconformismo en estos aspectos, pues sus expectativas no siempre se cumplen, debido a demoras, dificultades en el acceso y/o mala atención, aspectos que se analizaron en la evaluación del servicio.

Con base en la investigación, también se pudo establecer que la mayoría de los usuarios se encuentran satisfechos en aspectos como el horario de atención, la calidad de los productos y la ubicación del sitio de compra, pero muchos no se encuentran satisfechos con los servicios adicionales que se ofrecen (o no se ofrecen), la agilidad en la atención, el servicio de asesoría, garantía, postventa y el acceso al parqueadero. Al evaluar al personal que presta sus servicios en las grandes superficies y ponderar los resultados se determinó que el nivel de satisfacción de los clientes es del $58 \%$, un nivel bajo que refleja la insatisfacción de los clientes, pues según lo manifestado por ellos mismos y lo observado en el sitio de compra, no hay por parte del personal interés o compromiso para brindar a los clientes un servicio fácil de ubicar, profesional, ágil y efectivo. Por lo anterior se concluye que el servicio al cliente no se percibe como una ventaja competitiva en las grandes superficies evaluadas, debido a las constantes quejas de usuarios insatisfechos. En este aspecto fue incluida la evaluación del servicio que prestan los vigilantes, impulsadoras, mercaderistas, cajeros, empacadores, supervisores y personal de servicio al cliente. Los resultados de esta evaluación muestran que el nivel de satisfacción de los clientes en cuanto al servicio que reciben por parte de este personal es más bajo que el nivel de satisfacción con relación a los aspectos evaluados anteriormente, es preocupante conocer que cerca del $50 \%$ de los clientes se encuentran inconformes con el servicio que reciben por parte del personal de servicio al cliente, para quienes por la naturaleza de su labor, el cliente es su razón de ser.

También se observó un bajo nivel de satisfacción con relación al servicio de los supervisores quienes tienen poco contacto con el cliente y al parecer estos momentos de verdad no son satisfactorios para los usuarios. Es significativo el re- 
sultado que se obtiene al evaluar la satisfacción del cliente con relación al servicio que reciben por parte de vigilantes, impulsadores, mercaderistas, cajeros y empacadores, pues en ninguno de estos cargos se alcanza un $70 \%$ de satisfacción según los clientes encuestados. Es de esperarse que los clientes atribuyan a estos funcionarios fallas en la atención, demoras e inconformismo en general sobre lo cual sería importante profundizar de manera particular en cada establecimiento.

Al evaluar el servicio ofrecido en la oficina de servicio o atención al cliente, se observó que de los usuarios encuestados, solo el $40 \%$ conocía o había hecho uso de estos servicios, al indagar a este $40 \%$ se encontró que el cumplimiento de las expectativas de los clientes encuestados es del $88 \%$ y un $12 \%$ de no cumplimiento. La insatisfacción de los clientes se acentúa en aspectos como la agilidad en la respuesta, el interés del empleado en la situación del cliente y la efectividad en la solución. A través de las observaciones realizadas en las grandes superficies se confirmó que las oficinas de atención o servicio al cliente no siempre están abiertas o disponibles al público, por lo cual sería importante garantizar la atención del cliente de forma permanente.

Así mismo, al evaluar el servicio de asesoría, garantía y postventa, se determinó que solo la cuarta parte de los clientes consultados había hecho uso de estos servicios. Se observó que hay un alto porcentaje de satisfacción en los aspectos como el profesionalismo y la facilidad para ubicar el servicio, pero un menor grado de satisfacción por la falta de soluciones efectivas y agilidad en la respuesta, pues el cumplimiento de las expectativas de los clientes encuestados con relación a los servicios de asesoría, garantía y postventa fue del $83 \%$ y un $17 \%$ el no cumplimiento. También se determinó que existen amplias diferencias en el manejo de estos servicios entre las grandes superficies, pues mientras en algunas hay un espacio amplio y número adecuado de personas para atender los requerimientos de asesoría, garantía y/o servicio de postventa, en otras estos servicios se prestan en espacios reducidos, casi de manera informal y con escaso personal.

En general, se concluye que el nivel de satisfacción de los clientes con relación a los servicios que reciben en las grandes superficies es bueno pero también hay evidentes oportunidades de mejoramiento principalmente en lo relacionado al personal que tiene contacto con los clientes, así como en cuanto a los servicios adicionales y los procesos de asesorías, garantías y postventa, durante los cuales se debe dar un mejor manejo a la información, pues más de cien encuestados hicieron observaciones relacionadas con el servicio en las cajas de pago, la garantía, la atención al cliente, el horario, la oferta y calidad de los productos, la tarjeta de puntos, el servicio del personal, entre otros.

\section{RECOMENDACIONES}

Se recomienda identificar de manera particular en cada una de las grandes superficies, cuáles son los factores que determinan la satisfacción del cliente para luego evaluar el desempeño de la organización en función de estos factores. Esta evaluación permitirá detectar y corregir las fallas que se puedan estar presentando durante los ciclos del servicio para mejorar la competitividad.

Con relación al personal que atiende a los clientes, se sugiere capacitarlos y crear una cultura de servicio en todos los empleados incluyendo impulsadoras, mercaderistas, supervisores, vigilantes, etc., para que la organización se distinga por prestar un excelente servicio a sus clientes y para que los usuarios se sientan siempre bien atendidos independientemente de sus preferencias de compra, la cantidad de productos o la hora en la que decidan realizarlas.

Adicionalmente, se recomienda hacer seguimiento a las solicitudes de los clientes y dar respuesta con mayor celeridad a sus requerimientos, informar con claridad a los clientes cuáles son sus derechos y deberes, así como el cubrimiento de la garantía y el procedimiento para los reclamos dejando constancia escrita, pues se deben crear canales de comunicación claros y efectivos con los clientes a todo nivel y en todas direcciones, de tal forma que se pueda recuperar la confianza en la gestión interna, ya que muchos de los usuarios consultados durante el trabajo de campo, manifestaron que sus inquietudes, quejas y reclamos no eran escuchados ni solucionados.

\section{REFERENCIAS}

Albrecht, K . (1998) La revolución del servicio. Bogotá: Legis Editores.

Albrecht, K. \& Bradford, L. (1990). La excelencia en el servicio. Bogotá: Legis Editores.

Albrecht, K. \& Zenke, K. (1991). Gerencia del Servicio. Bogotá: Legis Editores.

Cortés, M. E. \& Iglesias León, M. (2004). Generalidades sobre Metodología de la investigación. México: Universidad Autónoma del Carmen.

Cuatrecasas, L. (2010). Gestión integral de la calidad. Barcelona: Editorial Profit.

Garvin, D. A. (1987). Competing on the Eight Dimensions of Quality: Harvard Business Review, November-December..

Ginebra, J. \& Arena de la Garza, R. (1991) Dirección por servicio, la otra calidad. México: Editorial Mc Graw-Hill. 
Horovitz J. (2000). La calidad del servicio: A la conquista del cliente. Madrid: Editorial McGraw-Hill.

Huete, L. M. (2001) Servicios y Beneficios, la fidelización de clientes y empleados, la inteligencia emocional en los negocios. Barcelona: Ediciones Deusto.

Ishikawa, K. (1994). ¿Qué es el control total de calidad? La Modalidad Japonesa. Bogotá: Editorial Norma.

Juran, J. M. (1993). Manual de Control de la Calidad IV Edición. España: Editorial Mc Graw Hill Interamericana.

Kotler P. G. A. (2003). Fundamentos de Marketing VI Edición. Bogotá: Editorial Pearson Educación.

Montecinos, J. (2009). Modelo de evaluaciones de experiencias de consumo. Madrid: Universidad Autónoma de Madrid.

NTC-ISO 8402 (1986). Vocabulario sobre la calidad. Bogotá: Icontec.

NTC-ISO 9000. (2001). Sistemas de gestión de la calidad. Fundamentos y vocabulario. Bogotá: Icontec.
NTC-ISO 9001. (2009). Sistemas de gestión de la calidad. Requisitos. Bogotá: Icontec.

Oliver, R. A. (1993). Conceptual model of service quality and service satisfaction. Compatible goals, different concepts. Advances in Services Marketing and Management, Vol. 2, 65-85.

Pedic, F. (2004). Medición de la satisfacción del cliente: Un manual para usuarios de la ISO 9001:2000. Bogotá: Editorial Standards.

Peralta, J. (2006). Rol de las expectativas en el juicio de satisfacción y calidad percibida del servicio. Límite, Revista de filosofía y psicología, vol 1, No. 14, pp. 195-214.

Sainz, J. M. (2001). La Distribución comercial: Opciones estratégicas. Madrid: Editorial Esic.

Serna, H. (2007). ¿Cómo medir la satisfacción de clientes? Bogotá: Universidad de los Andes.

Uribe, M. E. (2011). Gerencia del servicio. Bogotá: Ediciones de la $U$. 